\title{
AUTORREGULAÇÃO DA APRENDIZAGEM EM COMPUTAÇÃO COM APOIO DA METODOLOGIA SCRUM
}

\author{
Karen Selbach Borges/IFRS - Campus Porto Alegre \\ Márcia Amaral Corrêa de Moraes/IFRS - Campus Porto Alegre \\ Fabio Y. Okuyama/IFRS - Campus Porto Alegre
}

\begin{abstract}
RESUMO: O presente trabalho apresenta o relato da experiência de desenvolvimento de processos autorregulatórios de aprendizagem com uma turma de terceiro semestre do curso Superior em Tecnologia de Sistemas para Internet, do Instituto Federal de Educação, Ciência e Tecnologia do Rio Grande do Sul (IFRS) - campus Porto Alegre. Para tanto, foram utilizados recursos como o desenvolvimento de projetos acadêmicos apoiados pelo uso da metodologia Scrum e a avaliação baseada em rubricas. Pretende-se, através deste relato, instigar os leitores a uma reflexão sobre a necessidade da inovação nos meios educacionais, a fim de promover uma mudança de comportamento e atitude dos alunos em relação ao seu processo de aprendizagem.
\end{abstract}

PALAVRAS-CHAVE: Autorregulação da aprendizagem. Metodologias ágeis. Aprendizagem no ensino superior. Scrum. Educação profissional.

\begin{abstract}
This paper presents a self regulated learning experience carried on in an Information Technology undergraduate program, of Instituto Federal de Educação, Ciência e Tecnologia do Rio Grande do Sul (IFRS) - campus Porto Alegre. The studied population was composed by third semester students. We used resources such as the development of academic projects supported by the use of Scrum methodology and based on assessment rubrics. We intend, through this report, lead readers to reflect about the need for innovation in education, in order to promote behavior and attitude changes of students in relation to their learning process.
\end{abstract}

KEYWORDS: Self-regulation of learning. Agile methodologies. Learning in higher education. Scrum. Professional education.

\section{INTRODUÇÃO}

O trabalho é parte essencial da vida das pessoas. Desde o princípio, é nele que buscamos os meios para o nosso sustento e o de nossas famílias. A natureza do trabalho vem se modificando ao longo do tempo e, nesse contexto, o termo "capital humano", que, segundo Harbison (1974, p. 3 apud MANFREDI, 1999, p. 45), significa "processo de formação e incremento do número de pessoas que possuem as habilidades, educação e a experiência indispensáveis para o desenvolvimento político e econômico de um país”, tem ocupado lugar de destaque. O Brasil precisará, até 2015, de um adicional de 8 milhões de pessoas educadas e qualificadas para assumir funções cada vez mais sofisticadas (SALOMÃO; GIANINI, 2011). 
O governo brasileiro vem trabalhando para melhor qualificar a população de trabalhadores. Nesse sentido, foram criados os Institutos Federais de Educação, Ciência e Tecnologia (Ifs), que, segundo o ex-secretário de Educação Profissional e Tecnológica do Ministério da Educação (Setec), Eliezer Pacheco (2011, p. 27), “responderão de forma mais ágil e eficaz às demandas crescentes por formação de recursos humanos, difusão de conhecimentos científicos e suporte aos arranjos produtivos locais”. O Instituto Federal de Educação Ciência e Tecnologia, mais especificamente o campus Porto Alegre, oferece, além de cursos técnicos, quatro cursos de nível superior, dentre os quais destacamos o curso Superior de Tecnologia em Sistemas para Internet, que conta, atualmente, com três turmas em funcionamento no turno da manhã. Foi na turma de terceiro semestre, no ano de 2012, que se desenvolveu o experimento sobre autorregulação da aprendizagem realizado por meio de práticas oriundas da metodologia Scrum (SCHWABER; SUTHERLAND, 2013), aplicadas no desenvolvimento de projetos de sala de aula.

A Scrum é uma das metodologias ágeis utilizadas no desenvolvimento interativo e incremental de produtos, baseada no seguinte conjunto de princípios: (i) aceitar as incertezas; (ii) realizar constante planejamento; (iii) trabalhar como um time; (iv) manter equipes autogerenciáveis; (v) manter um ritmo de trabalho suportável; (vi) realizar entregas frequentes (SCHWABER; SUTHERLAND, 2013). Diante do exposto, destacamos que o objetivo deste trabalho é investigar a possibilidade de aplicação desses princípios em um processo de autorregulação da aprendizagem.

Conforme Rosário (2002, p. 39), o termo autorregulação pode ser definido como um "processo ativo no qual os sujeitos estabelecem os objetivos que norteiam a sua aprendizagem tentando monitorizar, regular e controlar as suas cognições, motivação e comportamentos com o intuito de os alcançar”. Assim, o processo de autorregulação envolve desenvolver autonomia, iniciativa, planejamento e organização, que, por sua vez, são fundamentais para garantir a aplicabilidade dos princípios da Scrum.

A título de organização, o presente artigo está estruturado da seguinte forma: na seção 1, analisaremos os aspectos relativos à autorregulação da aprendizagem; na seção 2, trataremos da metodologia do experimento realizado e, na seção 3, será apresentada a análise dos resultados obtidos e as considerações finais.

\section{AUTORREGULAÇÃO DA APRENDIZAGEM}

A proposta de trabalho apresentada neste artigo tem como alicerce o uso da ferramenta Scrum como formato de intervenção pedagógica voltada à realização de processos autorreguladores de aprendizagem por parte dos sujeitos envolvidos. Os alunos compreendidos nessa proposta foram orientados sobre como apreender e utilizar os recursos pessoais que lhes permitissem refletir sobre as suas aprendizagens e reforçar as suas competências para aprender. Conforme Lopes da Silva et al. (2004), somente na posse consciente, conhecedora e controlada de meios internos e externos, é que os sujeitos poderão exercer um papel ativo na construção dos seus saberes, na concretização das suas aspirações, na elaboração e direção dos seus objetivos intelectuais, afetivos, sociais e profissionais. É justamente nesse contexto, de posse sobre as próprias possibilidades, que a autorregulação entra em cena na vida dos acadêmicos do curso Superior em Tecnologia de Sistemas para Internet.

Autorregular-se significa viver processos de aprendizagem regulados pelo próprio 
aprendente. Tal regulação é em grande parte resultante da interação de conhecimentos, competências e motivações que são necessários ao planejamento, à organização, ao controle e à avaliação dos processos adotados e dos resultados atingidos numa determinada situação de ensino sistemático. Nesse sentido, a base do processo de autorregulação está na constituição de uma aprendizagem mais autônoma e pró-ativa, na qual o aprendente mobiliza as suas características pessoais e desenvolve estratégias assertivas para maximizar a sua aprendizagem nas mais diversas áreas da sua vida (ZIMMERMAN, 1998). As tomadas de consciência resultantes desse processo conduzem a um maior envolvimento motivacional e à aplicação de estratégias metacognitivas (ZIMMERMAN, 2008) em âmbitos diversos e mais amplos (da sala de aula para o mundo do trabalho, por exemplo).

O uso da metodologia Scrum neste contexto tem como um de seus objetivos, levar os estudantes a regularem suas aprendizagens. Conforme Montalvo e Torres (2004), os sujeitos que autorregulam suas aprendizagens comportam-se da seguinte forma:

- Conhecem e sabem aplicar uma série de estratégias cognitivas (de repetição, elaboração e organização) que os ajudam a entender, transformar, organizar, elaborar e recuperar a informação;

- Sabem como planejar, controlar e dirigir os seus processos mentais, articulandoos às suas metas pessoais (metacognição);

- Apresentam um conjunto de crenças motivacionais e emocionais adaptativas, tais como o sentido de auto-eficácia acadêmica, a adoção de metas de aprendizagem, o desenvolvimento de emoções positivas face às tarefas (por exemplo: satisfação e entusiasmo), assim como têm capacidade para controlá-las e modificá-las, ajustando-as às exigências da tarefa e da situação de aprendizagem concreta;

- Planejam e controlam o tempo e o esforço que necessitam imprimir às tarefas e sabem criar e estruturar ambientes favoráveis de aprendizagem, como por exemplo, encontrar um lugar adequado para estudar e procurar ajuda dos professores e colegas quando têm dificuldades;

- Na medida em que o contexto permite, mostram uma intenção mais forte em participar do controle e da regulação de tarefas acadêmicas, do clima e da estrutura da sala de aula (por exemplo: conhecimento dos critérios de avaliação, exigências da tarefa, planejamento dos trabalhos em sala de aula e organização de grupos de trabalho);

- São capazes de fazer uso de uma série de estratégias volitivas orientadas para evitar distrações externas e internas, para manter a concentração, o esforço e a motivação durante a realização das tarefas acadêmicas. (MONTALVO; TORRES, 2004, p. 3.).

A aprendizagem autorregulada pelo próprio aluno resulta da interação de variáveis pessoais (conhecimentos, competências e motivações) que proporcionam ao estudante o planejamento, a organização, o controle e a avaliação dos processos adotados, dos resultados atingidos e das variáveis contextuais, que o estimulam e lhe dão a oportunidade de agir de uma forma intencional e estratégica. Conforme Zimmerman (2000), os processos psicológicos implicados nas diversas fases da autorregulação, e que são constituintes da prática nesse artigo descrita, são os seguintes: fase de antecipação e preparação (antes), fase de execução e controle (durante) e fase de autorreflexão (depois). Na fase de antecipação, os desafios do estudante estão na análise da tarefa e na influência das crenças automotivacionais. Como formas de análise da tarefa, podem ser apontados o estabelecimento de objetivos, que se refere aos resultados específicos pretendidos com a realização de uma determinada tarefa acadêmica e o planejamento estratégico 
que se reporta à seleção de estratégias ou métodos de aprendizagem necessários para a obtenção dos objetivos estabelecidos. O planejamento e a seleção de estratégias de aprendizagem requerem, por sua vez, adaptações sucessivas devido às flutuações nos componentes do contexto em que o aprendente se situa.

Na fase de execução e controle volitivo são exigidos dois processos: o autocontrole e a automonitorização. O primeiro processo inclui as autoinstruções, as imagens mentais, a focalização da atenção, as estratégias utilizadas na realização das tarefas e a ajuda prestada aos aprendentes para se concentrarem nas tarefas e otimizarem os seus esforços. A automonitorização refere-se à atenção que o aluno dedica a aspectos específicos da sua realização, às condições que lhe estão subjacentes e aos efeitos produzidos. Facilmente se compreende que as estratégias de aprendizagem relacionadas com uma determinada tarefa ajudam o aluno a dividi-la nas suas partes essenciais, reorganizando-as num todo coerente. O segundo processo na fase de controle volitivo, a automonitorização, é considerado como crucial para a autorregulação porque informa o aluno acerca dos seus progressos e retrocessos face a um determinado critério de referência (por exemplo: as classificações acadêmicas, objetivos da disciplina definidos pelo professor e o sucesso acadêmico dos pares). Nesse modelo proposto por Zimmerman (2000), é possível diferenciar a automonitorização associada à autoavaliação, à implementação de estratégias e aos esforços para adaptá-las a partir dos resultados obtidos.

A última fase do ciclo de autorregulação, denominada fase da autorreflexão, envolve essencialmente dois processos: a autoavaliação e a autorreação. A autoavaliação refere-se à forma como o aprendente avalia os resultados obtidos, sendo influenciado por pensamentos como as atribuições ou padrões autoimpostos. A autorreação, por sua vez, positiva ou negativa, diz respeito à comparação da informação automonitorizada com um critério ou objetivo previamente definido. A existência desse objetivo, padrão, critério ou valor de referência serve como medida para avaliar a ação do sistema e orientar os processos de regulação.

Os objetivos que orientam a regulação são representações cognitivas de um acontecimento futuro e influenciam a motivação através de seis processos: a) dirigem a atenção e a ação para um alvo intencional, o que ajuda a pessoa a centrar-se na tarefa e a reunir os seus recursos para atingir o objetivo; b) mobilizam o esforço proporcionalmente à dificuldade da tarefa; c) promovem a persistência e o esforço ao longo do tempo; d) fornecem uma razão para continuar a trabalhar mesmo se a atividade não está se desenvolvendo a contento; e) promovem o desenvolvimento de planos e estratégias criativas para os atingir e f) fornecem um ponto de referência que dá informação sobre a qualidade do desempenho (ALDERMAN, 1999).

Segundo Pintrich (2000), os objetivos representam uma “orientação geral” para a tarefa que inclui, ela própria, uma série de crenças relacionadas com propósitos, competências, sucesso, capacidades, esforços, erros e critérios (LOPES DA SILVA et al., 2004). Essa teoria presume que os objetivos são de natureza cognitiva e acessíveis à pessoa. A questão é determinar quando e como se tornam conscientes e atuam de forma a influenciar a motivação e o comportamento antes, durante e após a realização de uma tarefa.

No âmbito da implementação de práticas de autorregulação, é importante destacar o papel das estratégias de aprendizagem que devem ser construídas pelos alunos. As estratégias de aprendizagem são sempre conscientes e intencionais, dirigidas para um objetivo diretamente relacionado com a aprendizagem. Elas são, portanto, diferentes das técnicas de estudo, que podem ser utilizadas de forma mais ou menos mecânica sem que, para a sua aplicação, exista um propósito de aprendizagem por parte de quem as utiliza (VEIGA SIMÃO, 2002). Isso significa que trabalhar 
com autorregulação envolve majoritariamente investir no desenvolvimento de estratégias de aprendizagem pelo aprendiz, de modo que consiga apropriar-se do seu processo de aprender, bem como colocar-se como autor do mesmo.

A autonomia do aprendente representa uma conquista efetiva desse tipo de proposta de ensino. Ela deve manifestar-se ao longo do ciclo de autorregulação, como o descrito nesse trabalho, e envolve necessariamente o uso de estratégias metacognitivas, principalmente as estratégias superiores de planejamento e de decisão dos componentes cognitivos que são atinentes às situações de resolução de problemas. Para tanto, o indivíduo necessita recorrer a uma espécie de monitorização do seu próprio pensamento (pensar sobre o pensar). Dessa forma, a metodologia Scrum foi utilizada nesse trabalho no sentido de promover o processo autorregulatório da aprendizagem junto aos acadêmicos envolvidos no presente estudo.

\section{METODOLOGIA}

A disciplina de Programação para Web I, ministrada pela primeira autora deste artigo, foi escolhida para a realização deste experimento por apresentar as seguintes características:

- Carga horária: 90 horas, distribuídas em 5 períodos semanais e consecutivos, o que permitiu um tempo maior para o desenvolvimento dos projetos em sala de aula.

- Conteúdo: programação Java para Web, que se considerou como sendo um conteúdo de alto nível motivacional, pois permite aos alunos o desenvolvimento de aplicações Web com maior nível de complexidade.

- Possibilidade de realização de um trabalho interdisciplinar: 12, dos 14 alunos, já haviam iniciado a implementação do projeto escolhido na disciplina de programação Java do semestre anterior. Além disso, 10 alunos estavam matriculados em Banco de Dados II e Engenharia de Software II, o que permitia efetivar a proposta de interdisciplinariedade.

Na primeira aula, foi apresentada ao grupo de alunos a proposta do trabalho a ser realizado e juntos, alunos e professora, definiram as formas de avaliação. A metodologia de trabalho acordada pode ser observada nas subseções a seguir.

\subsection{Aula dividida em períodos de teoria e momentos de prática aplicada}

Os encontros semanais de 5 períodos consecutivos foram organizados de modo a propiciar momentos para o desenvolvimento do conteúdo e tempo para os grupos discutirem o andamento dos trabalhos, distribuírem tarefas, trocarem ideias e esclarecerem dúvidas com a professora. Destaca-se a realização de reuniões rápidas, em que cada membro do grupo deveria responder as seguintes perguntas: 1) o que eu fiz desde o último encontro?; 2) o que vou fazer até o próximo?; 3) existem impedimentos (para minhas atividades)?. 


\subsection{Desenvolvimento de um projeto interdisciplinar}

A realização de um trabalho envolvendo a integração de diferentes disciplinas já era prevista no Projeto Pedagógico do Curso. Assim, em cada uma das disciplinas envolvidas, os grupos ficaram responsáveis pela elaboração de um produto:

- Levantamento de requisitos do sistema e sua modelagem na notação UML (Unified Modeling Language) foram desenvolvidos na disciplina de Engenharia de Software II;

- Implementação do sistema foi realizada na disciplina de Programação para Web I;

- Modelagem, implementação e programação do banco de dados, na disciplina de Banco de Dados II.

\subsection{Utilização de práticas ágeis da metodologia Scrum}

Sabe-se que o uso de metodologias ágeis no desenvolvimento de projetos de Sistemas de Informação é prática cada vez mais comum nas empresas da área de Tecnologias da Informação (TI). Esse cenário foi apresentado aos alunos que concordaram em adotar algumas práticas da metodologia Scrum com vistas a obterem melhor organização na divisão de tarefas e melhor acompanhamento na evolução do desenvolvimento do projeto. Dentro da prática da Scrum, utilizase o conceito de sprint, em que, de acordo com Schwaber e Sutherland (2013), trata-se de um ciclo do processo de desenvolvimento de um mês ou menos, durante o qual uma versão incremental potencialmente utilizável do produto é desenvolvida. Os papéis envolvidos no Scrum são: scrum master, team leader e team member. O scrum master seria, de modo geral, o gerente de projeto, o team leader o líder da equipe de programação e os demais teriam o papel de team member.

Assim, a turma foi divida em grupos de quatro alunos. Cada grupo definiu o projeto a ser desenvolvido conforme seus interesses pessoais. Os alunos se revezaram no papel de team leader a cada quatro semanas, período compreendido pelo sprint. Para o planejamento dos sprints e acompanhamento da evolução dos projetos, foi utilizada a ferramenta ScrumMe. Nessa ferramenta, os sprints foram descritos em função de histórias e tarefas, que eram distribuídas entre os membros do grupo.

\subsection{Avaliação formativa}

Considerando as práticas da Scrum, a professora assumiu o papel de scrum master. Coube a ela, então, conduzir as reuniões de final de sprint, ajudar o grupo a avaliar o andamento dos projetos, reorganizar tarefas e tentar resolver conflitos e impedimentos do grupo. Foi desenvolvido, junto com os alunos, um instrumento de acompanhamento, conforme Figura 1 a seguir: 


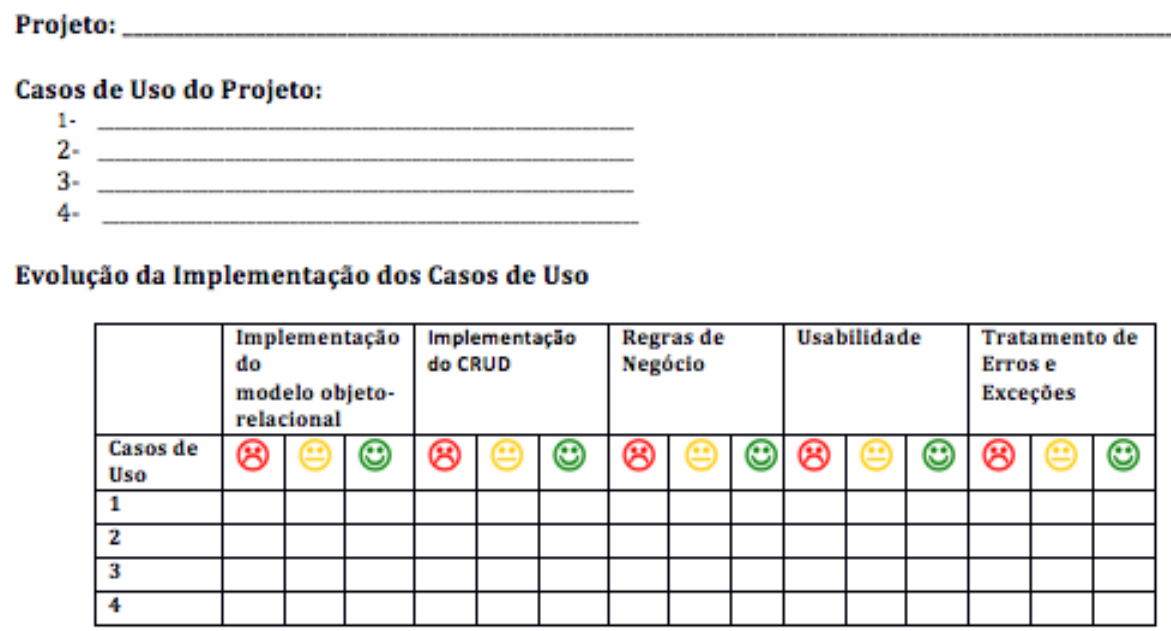

$\underline{+}$

Evolução dos Sprints

\begin{tabular}{|l|l|l|l|l|}
\hline & Sprint1 & Sprint2 & Sprint3 & Sprint4 \\
\hline Team lider & & & & \\
\hline Data & & & & \\
\hline Objetivos & & & & \\
\hline Conclusão & & & & \\
\hline
\end{tabular}

Figura 1: ficha de acompanhamento da evolução dos projetos.

Foi combinado com os alunos que, para aprovação na disciplina, não poderia haver marcações vermelhas em suas fichas. Adicionalmente, os alunos seriam avaliados individualmente pela sua participação nos projetos (envolvimento nas tarefas e atenção ao cumprimento dos prazos determinados pelo próprio grupo) e, finalmente, seria realizada uma autoavaliação no segundo e no último sprint. Para tanto, foi desenvolvido um instrumento adaptado a partir das rubricas propostas por Franker (2007). O Quadro 1, a seguir, apresenta uma amostra de tal instrumento.

Quadro 1: Rubricas para autoavaliação de alunos em trabalhos colaborativos.

\begin{tabular}{|c|c|c|c|c|}
\hline Categoria & Excelente & Muito Bom & Bom & Insatisfatório \\
\hline & 3 pontos & 2 pontos & 1 ponto & 0 pontos \\
\hline $\begin{array}{l}\text { Foco na tarefa } \\
\text { e participação }\end{array}$ & $\begin{array}{l}\text { Permanece } \\
\text { focado na tarefa e } \\
\text { no que precisa ser } \\
\text { feito. Pró-ativo. }\end{array}$ & $\begin{array}{l}\text { Concentra-se na } \\
\text { tarefa e no que } \\
\text { precisa ser feito a } \\
\text { maior parte do } \\
\text { tempo. Outros } \\
\text { membros do } \\
\text { grupo podem } \\
\text { contar com essa } \\
\text { pessoa. }\end{array}$ & $\begin{array}{l}\text { Concentra-se na } \\
\text { tarefa e no que } \\
\text { precisa ser feito } \\
\text { algum tempo. } \\
\text { Outros membros do } \\
\text { grupo devem, por } \\
\text { vezes, lembrar essa } \\
\text { pessoa de continuar } \\
\text { a tarefa. }\end{array}$ & $\begin{array}{l}\text { Raramente se } \\
\text { concentra na } \\
\text { tarefa e no que } \\
\text { precisa ser feito. } \\
\text { Permite que } \\
\text { outros façam o } \\
\text { trabalho. }\end{array}$ \\
\hline
\end{tabular}




\begin{tabular}{|l|l|l|l|l|}
\hline $\begin{array}{l}\text { Resolução de } \\
\text { problemas }\end{array}$ & $\begin{array}{l}\text { Busca ativamente } \\
\text { e sugere soluções } \\
\text { para os } \\
\text { problemas. }\end{array}$ & $\begin{array}{l}\text { Eventualmente } \\
\text { busca novas } \\
\text { soluções. }\end{array}$ & $\begin{array}{l}\text { Não sugere ou } \\
\text { refina soluções, } \\
\text { mas está disposto a } \\
\text { explmente refina } \\
\text { experimentar } \\
\text { soluções suges } \\
\text { pougeridas outros. } \\
\text { outros. }\end{array}$ & $\begin{array}{l}\text { Não tenta } \\
\text { resolver } \\
\text { problemas ou } \\
\text { ajudar os outros a } \\
\text { resolver } \\
\text { problemas. }\end{array}$ \\
\hline
\end{tabular}

\section{ANÁLISE DOS RESULTADOS}

O acompanhamento do projeto foi feito ao final de cada sprint. Na Tabela 1, é possível observar a evolução dos projetos.

Tabela 1: Evolução dos sprints.

\begin{tabular}{c|c|c|c|c} 
Grupo & $\begin{array}{c}\text { Sprint 1 } \\
25 / 10 / 2012\end{array}$ & $\begin{array}{c}\text { Sprint 2 } \\
22 / 11 / 2012\end{array}$ & $\begin{array}{c}\text { Sprint 3 } \\
20 / 12 / 2012\end{array}$ & $\begin{array}{c}\text { Sprint 4 } \\
17 / 01 / 2012\end{array}$ \\
\hline GCPark & $72 \%$ & $20 \%$ & $13 \%$ & $70 \%$ \\
\hline Webcar & $100 \%$ & $100 \%$ & $100 \%$ & $63 \%$ \\
\hline Siatco & $100 \%$ & $81 \%$ & $50 \%$ & $80 \%$ \\
\hline Matext & $66 \%$ & $0 \%$ & $42 \%$ & $100 \%$
\end{tabular}

Observa-se que, na média, o primeiro sprint foi o que apresentou melhor rendimento (84,5\%). Isso se deu graças ao alto nível motivacional dos alunos que estavam ansiosos pela oportunidade de desenvolver projetos próprios com maior nível de complexidade. Os sprints 2 e 3 apresentaram resultados medianos, justificados pelos alunos não como falta de interesse na disciplina, mas pelo acúmulo de atividades como trabalhos e provas de outras disciplinas e atividades profissionais, uma vez que a maioria dos alunos já estava inserida no mercado de trabalho. O último sprint apresenta melhoras no desempenho que, segundo os alunos, aconteceu graças à maior disponibilidade de tempo livre para os projetos, uma vez que parte do sprint 4 aconteceu durante o período de recesso de fim de ano.

Foi possível verificar o grau de participação de cada membro do grupo, graças ao uso da ferramenta ScrumMe, através da qual era possível gerar gráficos de acompanhamento do projeto. A Figura 2 mostra, a título de exemplo, o gráfico do grupo denominado Webcar. A ferramenta mostra as atividades realizadas, em andamento e a fazer. Além disso, exibe a proporção de atividades realizadas para cada membro do time. A figura foi editada para omitir os nomes dos alunos envolvidos. 

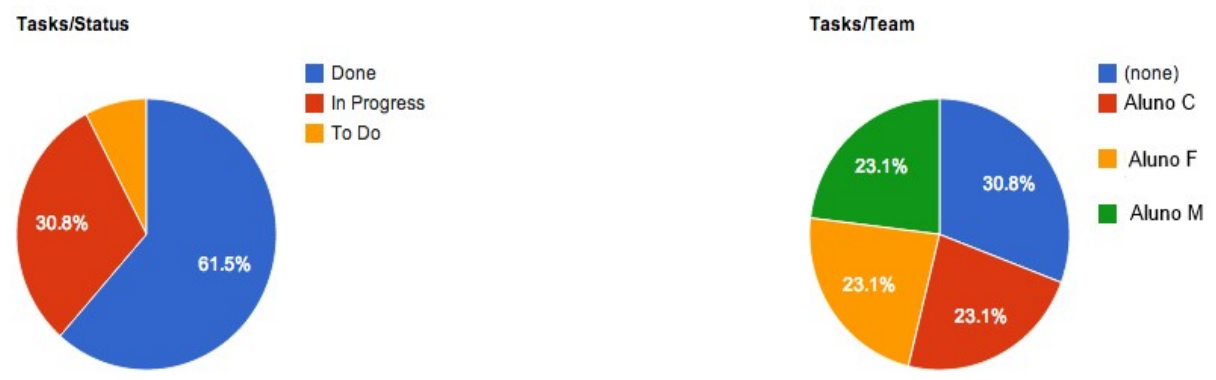

Figura 2: gráficos de andamento do sprint e de distribuição de atividades.

Em relação à autoavaliação, os alunos deram alguns depoimentos interessantes e que ajudam a corroborar a ideia de que é importante trabalhar não apenas competências técnicas, mas também as competências atitudinais necessárias ao "saber fazer, saber agir e saber ser", que Manfredi (1999, p. 18) defende como sendo o perfil ideal de profissional para empresas inovadoras. Esses depoimentos, juntamente com comentários da professora da disciplina são apresentados no Quadro 2.

Quadro 2: Depoimento dos alunos e comentários da professora

\begin{tabular}{|c|c|}
\hline Depoimento do Aluno & Visão da professora \\
\hline $\begin{array}{l}\text { "Ao final da cadeira percebi a mudança na minha postura } \\
\text { com relação ao projeto, procurei me policiar e focar no } \\
\text { projeto". }\end{array}$ & $\begin{array}{l}\text { Esta aluna mostrou um nível de } \\
\text { comprometimento com o trabalho nunca antes } \\
\text { observado. }\end{array}$ \\
\hline $\begin{array}{l}\text { "Durante o desenvolvimento do projeto houve grande } \\
\text { oportunidade de crescimento de aprendizagem através de } \\
\text { situações adversas e dificuldades encontradas". }\end{array}$ & $\begin{array}{l}\text { Este aluno pesquisou e aplicou soluções que } \\
\text { não faziam parte da ementa da disciplina. }\end{array}$ \\
\hline $\begin{array}{l}\text { "De modo geral minha auto avaliação diminuiu, pois } \\
\text { percebi que fiz somente o que era necessário para que o } \\
\text { software funcionasse." }\end{array}$ & $\begin{array}{l}\text { Essa aluna, transferida de outra instituiç̧ão de } \\
\text { ensino, inicialmente apresentou dificuldade } \\
\text { para iniciar o trabalho, mas seu } \\
\text { comportamento de liderança foi decisivo para } \\
\text { a conclusão do projeto. }\end{array}$ \\
\hline $\begin{array}{l}\text { "Antes me dei nota } 3 \text { no item foco na tarefa e participação. } \\
\text { Agora mudei para } 2 \text { porque reconsiderei que ainda posso } \\
\text { melhorar para me tornar um valoroso membro da equipe } \\
\text { que "incentiva e apoia os outros no grupo".” }\end{array}$ & $\begin{array}{l}\text { Esse aluno, apesar do excelente desempenho } \\
\text { técnico, percebeu que precisava melhorar } \\
\text { aspectos de comportamento e atitude. }\end{array}$ \\
\hline
\end{tabular}




\section{CONSIDERAÇÕES FINAIS}

Esta experiência trouxe para a sala de aula uma proposta inovadora para o desenvolvimento do processo autorregulatório dos alunos. Os alunos foram envolvidos em todas as etapas da disciplina: no planejamento, ao participarem da construção da metodologia adotada; na execução, ao se envolverem com a realização das atividades propostas e na avaliação, ao fazerem uma reflexão sobre o seu desempenho e da professora na condução da disciplina.

A professora, no papel de scrum master, foi responsável por ajudar os alunos na elaboração da relação de histórias, distribuição de tarefas ao longo dos sprints, busca de recursos para solução de problemas e acompanhamento do andamento dos trabalhos. Assim, o professor ultrapassou a função de "transmissor do conhecimento" para atuar também como orientador e conselheiro dos grupos. Essa mudança de perfil é defendida por Perrenoud (2007, p. 107), que sugere que o professor do século XXI deva ser “(i) organizador de uma pedagogia construtivista; (ii) garantia do sentido dos saberes; (iii) criador de situações de aprendizagem; (iv) administrador da heterogeneidade; (v) regulador dos processos e percursos de formação”. O autor acrescenta ainda a necessidade de uma postura reflexiva e crítica.

Ao envolver o Scrum, como forma de gerenciar o andamento dos projetos, foi possível observar, como benefício do uso de metodologias ágeis na educação, o trabalho colaborativo, o compromisso, a responsabilidade individual, a socialização de experiências, o compartilhamento de conhecimentos e a autorregulação. Esta última é compreendida em um sentido mais amplo, envolvendo não apenas os processos de aprendizagem, mas também o gerenciamento do tempo, a priorização de tarefas e a mudança de atitudes.

A forma de avaliação também influenciou no processo de autorregulação. Segundo Haydt (2000, p. 59 apud COSTA, 2012, p. 43) “cabe justamente à avaliação verificar em que medida os objetivos estão realmente sendo alcançados, para ajudar o aluno a avançar na aprendizagem”. Dessa forma, a avaliação foi utilizada como instrumento para acompanhar o crescimento do aluno, de forma processual e contínua, e propor ajustes e reajustes no processo de aprendizagem.

\section{REFERÊNCIAS}

ALDERMAN, M. K. Motivation for Achievement: Possibilities for Teaching and Learning. Mahwah, NJ: Lawrence Erlbaum Associates, 1999.

COSTA, A. M.; SÁ, EDILANE M.; NUNES, E. S. Avaliação Significativa: desafios e perspectivas para o ensino superior. 2012. Disponível em: <http://www.webartigos.com/artigos/avaliacaosignificativa-desafios-e-perspectivas-para-o-ensino-superior/98644/\#ixzz2Efav5vsX $>$. Acesso em: dez. 2012.

FRANKER, K. Collaboration Rubric. 2007. Disponível em: $<$ http://www2.uwstout.edu/content/profdev/rubrics/secondaryteamworkrubric.html $>$. Acesso em: out. 2012. 
HARBISON, F. H. Mão-de-obra e desenvolvimento econômico: Problemas e estratégia. In: PEREIRA, L. (Org.). Desenvolvimento, trabalho e educação. $2^{\mathrm{a}}$. ed. Rio de Janeiro: Zahar, 1974.

HAYDT R. C. Avaliação do processo ensino-aprendizagem. 6a. ed. São Paulo: Ática, 2000.

LOPES DA SILVA, Adelina et al. Aprendizagem auto-regulada pelo estudante: perspectivas psicológicas e educacionais. Porto: Porto Editora, 2004.

MANFREDI, S. M. Trabalho, qualificação e competência profissional - das dimensões conceituais e políticas. Educ. Soc.[online], 1999. Disponível em: <http://www.scielo.br/scielo.php?pid=S010173301998000300002\&script=sci abstract\&tlng=pt>. Acesso em: set. 2012.

MONTALVO, F.; TORRES, M. El aprendizaje autorregulado: presente y futuro de la investigación. Revista Electrónica de Investigación Psicoeducativa, 2004, p. 01-34 Disponível em: <http://www.investigacion-psicopedagogica.org/revista/articulos/3/espannol/Art 3 27.pdf $>$. Acesso em: abr. 2013.

PACHECO, E. Institutos Federais uma revolução na educação profissional e tecnológica. Moderna: São Paulo, 2011.

PERRENOUD, Philippe. As competências para ensinar no século XXI: a formação de professores e o desafio da avaliação. Porto Alegre: Artmed, 2007.

PINTRICH, P. R. The role of goal orientation in self-regulated learning. In: BOEKAERTS, M.; PINTRICH, P.; ZEIDNER, M. (Eds.), Handbook of Self-Regulation. New York: Academic Press, 2000, p. 01-09.

ROSARIO, P. Estórias sobre o estudar, histórias para estudar: narrativas autorregulatórias na sala de aula. Porto, Porto Editora, 2002.

SALOMÃO, A.; GIANINI, T. Um país em busca de gente para trabalhar. Revista Exame, abr. 2011. Disponível em: < http://exame.abril.com.br/revista-exame/edicoes/0989/noticias/um-pais-em-buscade-gente-2>. Acesso em: jul. de 2013.

SCHWABER, K. SUTHERLAND, J. The Scrum Guide - The Definitive Guide to Scrum: The Rules of the Game. 2013. Disponível em: <http://www.scrumguides.org/>. Acesso em: ago. De 2013.

VEIGA SIMÃO, A. M. A aprendizagem estratégica - Uma aposta na autoregulação. Coleção Curricular No 2. Lisboa: Ministério da Educação, 2002.

ZIMMERMAN, B. J. Academic Studying and Development of Personal Skill: a Self-Regulatory Perspective. Educational Psychologist, 33, 1998, p. 73. Disponível em: $<$ http://www2.sunysuffolk.edu/benharm/ed\%20psych/Web\%20Articles/Academic\%20studying \%20a\%20self\%20regulatory\%20perspective\%20full\%20article.pdf>. Acesso em: abr. 2013.

ZIMMERMAN, B. J. Attaining self-regulation: a social cognitive perspective. In: BOEKAERTS, M.; PINTRICH, P.; ZEIDNER, M. (Eds.), Handbook of Self-Regulation. New York: Academic Press, 2000, p. 01-27. 
ZIMMERMAN, B. J. Investigating self-regulation and motivation: historical background, methodological developments, and future prospects. American Educational Research Journal, 45, no. 1, 2008, p. 146-161. 